\title{
Aberration Correction System Using Segmented Detector in STEM
}

\author{
Y. Kohno ${ }^{1}$, H. Sawada ${ }^{1}$ and N. Shibata ${ }^{2}$ \\ 1. JEOL Ltd., 3-1-2 Musashino, Akishima, Tokyo 196-8558, Japan \\ 2. Institute of Engineering Innovation, School of Engineering, the University of Tokyo, Tokyo 116-0013, \\ Japan
}

Precise aberration measurement is essential for automated aberration correction systems, since it is one of determining factors in the performance of the automatic correction. Several aberration measurement methods for STEM are now in use, such as analyzing the Ronchigrams from an amorphous specimen, the probe form estimated from images of particles and so on. We cannot, however, observe the target specimen images immediately after the automatic aberration correction using these existing methods, since we have to change the illuminating apertures and exchange the specimen or the field of view. Moreover, we have to finally tune the aberrations (defocus, astigmatism or coma) manually during these procedures.

To avoid these processes, we need to estimate the aberrations from the images of the target specimens. There is a method of aberration measurements with the target specimen using image shifts of the BF STEM images detected with different azimuth and radial detection angles. This method is basically equivalent with defocus correction using an image wobbler, which is used in BF TEM, if we apply the reciprocal theory. If the geometrical aberrations of the illuminating system exist, the electron beam at the specimen surface does not focus in one point and have blurred distribution depending on the different incident angles and aberrations, resulting in the image shifts in the BF STEM image. For example, the simple defocus results in the circular image shifts with the same radial angle and various azimuth angles, and two fold astigmatism results in the ellipsoidal image shifts with the same radial angle and various azimuth angles. In this way, we can estimate the aberrations from the shifts of the BF STEM images. This method with a single detector was proposed years ago [1], but is not commonly used. This is partly due to the long image acquisition time of multiple images having different azimuth and radial detection angles and the measurement errors arise from the image drift during the acquisition. These are unavoidable for normal STEM detection systems, which controls the detection angle by deflectors in the image forming system and acquire only one image at a time. With a segmented detector, where each detector has different azimuth and radial detection angles, a set of images with different incident angles can be acquired simultaneously without image drifts.

We developed a new automatic aberration correction system using a segmented detector, which is composed of 16 multiple STEM detectors and is able to detect 16 images with different detection angles simultaneously [2]. The segmented detector is attached to the JEM-ARM300F with the STEM corrector system. The aberration correction module, embedded in the detector control system, calculates aberration coefficients from the relative image shifts of the acquired images. The module also communicates with the STEM corrector system through an ethernet, and the system controls the aberrations. This configuration realizes the automatic aberration correction on the target specimen using the segmented detector.

Figure 1 shows the Ronchigrams before and after the automatic aberration correction processes. We intentionally introduced defocus, 2-fold astigmatism, coma and 3-fold astigmatism before the processes. 
After the correction of these aberrations, the residual 6-fold astigmatism clearly appears in the Ronchigram. In this case, we use 8 BF STEM images acquired simultaneously, which are enough to calculate up to $2^{\text {nd }}$ order aberration coefficients. One loop takes about 10 seconds, which includes acquiring images, calculating aberrations and giving feedback to the corrector system. Figure 2 shows the dark field STEM images of gold particles on carbon thin film before and after the processes. These images show that the system can cope with large aberrations and that the measurement accuracy is high enough for high resolution imaging.

\section{References}

[1] O. L. Krivanek et al, Ultramicroscopy. 78 (1999) 1

[2] N. Shibata et al, J. Electron Microsc. 59 (2010) 473

[3] We thank Prof. Y. Ikuhara, the University of Tokyo for his continuous support. This development was supported by SENTAN, JST.
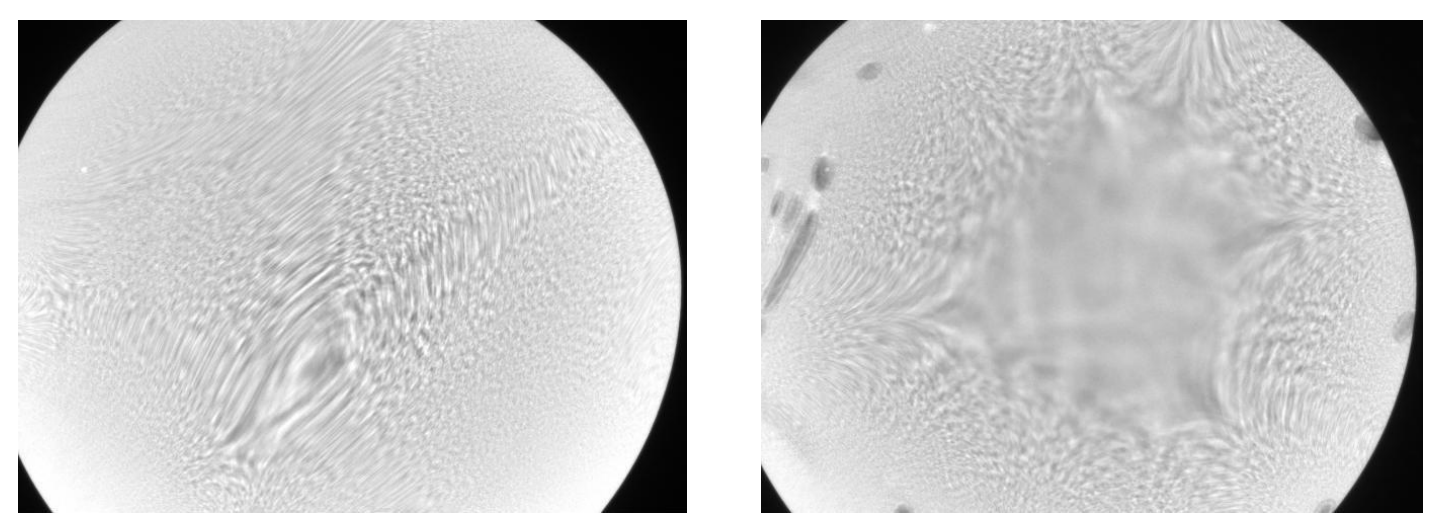

Figure 1. The Ronchigrams before (left) and after (right) the automatic aberration correction processes. Defocus, 2-fold astigmatisms, coma and 3-fold astigmatisms are intentionally introduced in the left Ronchigram. These are corrected in the right one.
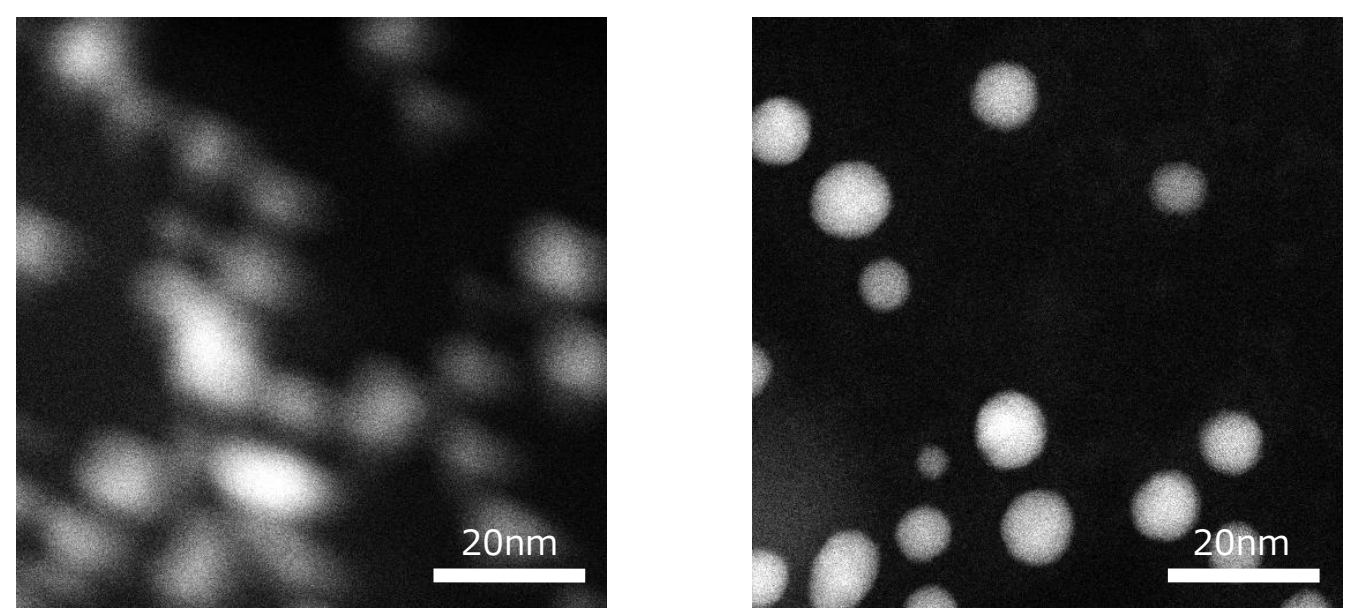

Figure 2. The dark field STEM images of gold nanoparticles on a carbon thin film. The left image was acquired before the processes and the right one is acquired after them. 